\title{
Intermediate Band Solar Cell with Extreme Broadband Spectrum Quantum Efficiency
}

\author{
A. Datas, E. López, I. Ramiro, E. Antolín, A. Martí, and A. Luque \\ Instituto de Energía Solar, Universidad Politécnica de Madrid, ETSI Telecomunicación, Ciudad Universitaria s/n, \\ 28040 Madrid, Spain
}

R. Tamaki, Y. Shoji, T. Sogabe, and Y. Okada

Research Center for Advanced Science and Technology, The University of Tokyo, 4-6-1 Komaba, Meguro-ku, Tokyo 153-8904, Japan

(Received 30 December 2014; revised manuscript received 25 February 2015; published 16 April 2015)

We report, for the first time, about an intermediate band solar cell implemented with InAs/AlGaAs quantum dots whose photoresponse expands from 250 to $~ 6000 \mathrm{~nm}$. To our knowledge, this is the broadest quantum efficiency reported to date for a solar cell and demonstrates that the intermediate band solar cell is capable of producing photocurrent when illuminated with photons whose energy equals the energy of the lowest band gap. We show experimental evidence indicating that this result is in agreement with the theory of the intermediate band solar cell, according to which the generation recombination between the intermediate band and the valence band makes this photocurrent detectable.

Intermediate band solar cells (IBSC) were proposed [1] as a means to exceed the efficiency of single gap solar cells thanks to the absorption of below-band gap energy photons. Intermediate band materials are characterized by the existence of a set of electronic states, named intermediate band (IB), within the semiconductor band gap $E_{G}$, splitting this in two sub-band gaps, $E_{L}$ and $E_{H}$, with $E_{L}$ the subband gap with the lowest energy. Because of this IB, belowband gap energy photons can pump electrons from the valence band (VB) to the IB, photons labeled 2 in Fig. 1(a), and from the IB to the conduction band (CB), photons labeled 3 in Fig. 1(a). The conversion efficiency limit of these cells is $63.2 \%$ and can be extended beyond $80 \%$ if incorporated in a multi-IB structure [2].

Since the concept emerged, several approaches have been proposed for its implementation. These approaches can be divided into those based in (a) quantum dots (QD IBSC) [3] [see Fig. 1(b)], (b) highly mistmached alloys [4], and (c) the insertion of selected impurities at high concentrations [5]. An exhaustive review detailing the experimental achievements by each of the approaches has been recently published [6]. These achievements, emphasizing the ones that are relevant for the discussion of this Letter and belonging to the QD IBSC category, can be summarized as follows.

Quantum efficiency measurements soon demonstrated that below-band gap energy photons, with energy enough to pump electrons from the $\mathrm{VB}$ to the $\mathrm{IB}$, were able to produce photocurrent [7]. However, this was not enough proof that QD IBSCs had the potential to exceed the efficiency of single gap solar cells, the reason relying on the mechanism promoting an electron from the IB to the CB. If this mechanism was thermal pumping, the additional subband gap photocurrent did not imply additional electrical work [8]. On the contrary, if this mechanism was due to the absorption of a second photon, additional electrical work was possible. It was in 2006 [9] that, when illuminating QD IBSC's simultaneously with low energy photons, capable only of pumping electrons from the IB to the $\mathrm{CB}$, and photons with energy capable of pumping electrons from the $\mathrm{VB}$ to the IB, it was demonstrated that electrons could be pumped from the IB to the $\mathrm{CB}$ thanks to photon absorption. Recently, the IB to $\mathrm{CB}$ transition has been spectrally resolved $[10,11]$ as a function of the photon energy. In addition, by measuring QD IBSCs under concentrated light, it has been found that the IB does not limit the open-circuit voltage of the QD IBSC [12]. The impact of QD doping on light absorption has also been explored $[13,14]$. Despite all these experimental results, a direct measurement of the photocurrent produced by the cell

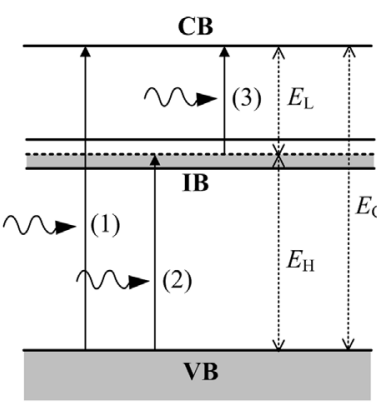

(a)

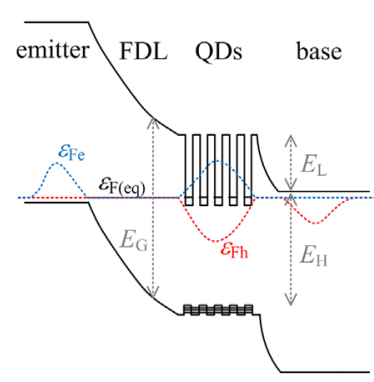

(b)
FIG. 1 (color online). (a) Simplified band gap diagram of an IB material showing the three relevant optical transitions and (b) idealized band diagram of a QD IBSC in short-circuit conditions illuminated by white light and considering finite carrier mobility. $\epsilon_{F e}$ and $\epsilon_{F h}$ are the quasi-Fermi energies for electrons and holes in the $\mathrm{CB}$ and $\mathrm{VB}$, respectively. 


\begin{tabular}{|c|c|}
\hline \multicolumn{2}{|c|}{$\mathrm{Cr} / \mathrm{Au}$} \\
\hline \multicolumn{2}{|c|}{$30 \mathrm{~nm} \mathrm{GaAs}\left(\mathrm{N}_{\mathrm{A}}=10^{19} \mathrm{~cm}^{-3}\right)$} \\
\hline $30 \mathrm{~nm}$ & $\mathrm{Al}_{0.8} \mathrm{Ga}_{0.2} \mathrm{As}\left(\mathrm{N}_{\mathrm{A}}=2 \times 10^{18} \mathrm{~cm}^{-3}\right)$ \\
\hline $200 \mathrm{~nm}$ & $\mathrm{Al}_{0.2} \mathrm{Ga}_{0.8} \mathrm{As}\left(\mathrm{N}_{\mathrm{A}}=10^{18} \mathrm{~cm}^{-3}\right)$ \\
\hline $400 \mathrm{~nm}$ & $\mathrm{Al}_{0.2} \mathrm{Ga}_{0.8} \mathrm{As}\left(\mathrm{N}_{\mathrm{D}}=10^{16} \mathrm{~cm}^{-3}\right)$ \\
\hline $25 \mathrm{~nm}$ & $\mathrm{Al}_{0.2} \mathrm{Ga}_{0.8} \mathrm{As}$ (intrinsic) \\
\hline \multicolumn{2}{|c|}{$\bullet \bullet \bullet \bullet \bullet \bullet \bullet \bullet$ InAs QDs (1e/QD) $\bullet \bullet \bullet \bullet \bullet \bullet \bullet \bullet$} \\
\hline $100 \mathrm{~nm}$ & $\mathrm{Al}_{0.2} \mathrm{Ga}_{0.8} \mathrm{As}$ (intrinsic) \\
\hline $500 \mathrm{~nm}$ & $\mathrm{Al}_{0.2} \mathrm{Ga}_{0.8} \mathrm{As}\left(\mathrm{N}_{\mathrm{D}}=10^{17} \mathrm{~cm}^{-3}\right)$ \\
\hline $100 \mathrm{~nm}$ & $\mathrm{Al}_{0.8} \mathrm{Ga}_{0.2} \mathrm{As}\left(\mathrm{N}_{\mathrm{D}}=1.5 \times 10^{17} \mathrm{~cm}^{-3}\right)$ \\
\hline & GaAs substrate $\left(\mathrm{N}^{+}\right)(100)$ \\
\hline
\end{tabular}

(a)

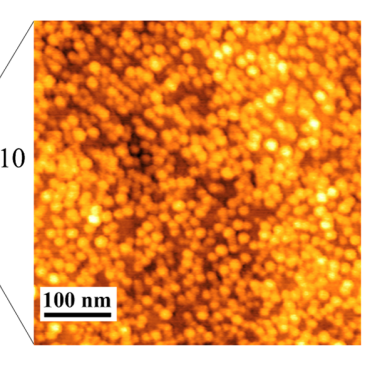

(b)
FIG. 2 (color online). (a) Layer structure of the QD IBSC under study and (b) AFM image of the QDs.

when illuminated solely with low energy photons, only capable of pumping electrons from the IB to the $\mathrm{CB}$, was missed. In this respect, this Letter provides this direct measurement for the first time.

The structure of the QD IBSC used in the experiments, consisting of InAs QDs inside an AlGaAs host, is detailed in Fig. 2(a). The structure was fabricated by MBE at growth temperature of $460^{\circ} \mathrm{C}$ and growth rates of $0.045 \mathrm{ML} / \mathrm{s}$ (InAs QDs) and $1 \mu \mathrm{m} / \mathrm{h}$ (AlGaAs barriers). As seen from AFM characterization [Fig. 2(b)], the mean diameter, height, and QD sheet density are $20.5 \mathrm{~nm}, 1.2 \mathrm{~nm}$, and $2.7 \times 10^{11} \mathrm{~cm}^{-2}$, respectively. Next to the front $p$ emitter an $n$-type "field damping layer" (FDL) [15] has been included. The doping and thickness of this layer have been calculated to sustain most of the depletion region so that the QDs are located within a flatband potential region [Fig. 2(b)]. This has the benefit of (i) preventing electrons in the IB from tunneling to the CB [16] and (ii) making it possible for the silicon doping of the QDs to partially fill the IB with electrons [15]. Partially filling the IB is important to provide it with both empty states to receive electrons from the VB as well as filled states to supply

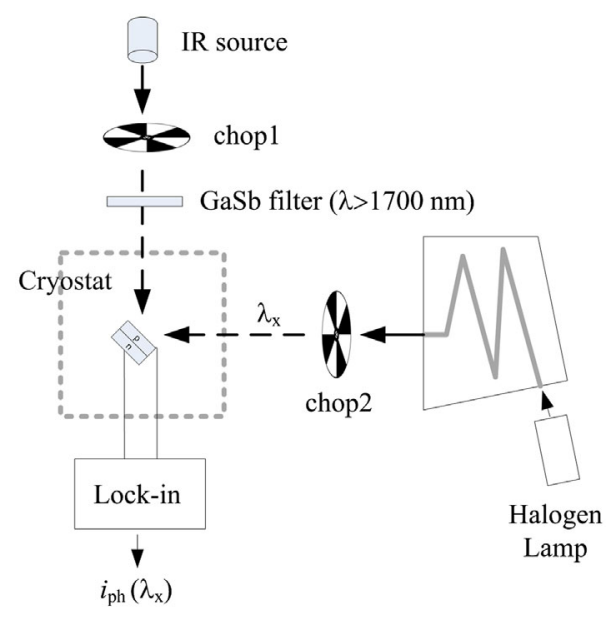

FIG. 3 (color online). Experimental setup.

electrons to the $\mathrm{CB}$. The QD IBSC semiconductor structure was processed into solar cell devices of an area $0.0314 \mathrm{~cm}^{-2}$ by using conventional photolithographic techniques.

The QD IBSC described above has been characterized using the experimental setup of Fig. 3, which comprises two light sources: (i) monochromatic (MC) and (ii) broadband IR. The MC light (photon wavelength of $\lambda_{x}$ ) is produced with a NewPort Cornerstone 7400 monochromator equipped with a halogen lamp and bandpass filters that avoid illuminating the cell with monochromator secondaries. The broadband IR light is produced by a steady state blackbody IR emitter filtered with a GaSb wafer $\left(\lambda_{\text {pass }}>1700 \mathrm{~nm}\right)$. The sample is located within a closed cycle He-gas cryostat and the output photocurrent $\left(i_{\mathrm{ph}}\right)$ is acquired with a lock-in amplifier synchronized to the frequency of either chopper 1 or 2 . All the photocurrent measurements presented in this Letter were carried out using a chopper frequency of $330 \mathrm{~Hz}$.

In a first experiment, the cell short-circuit photocurrent is measured under chopped MC light illumination (chopper 1

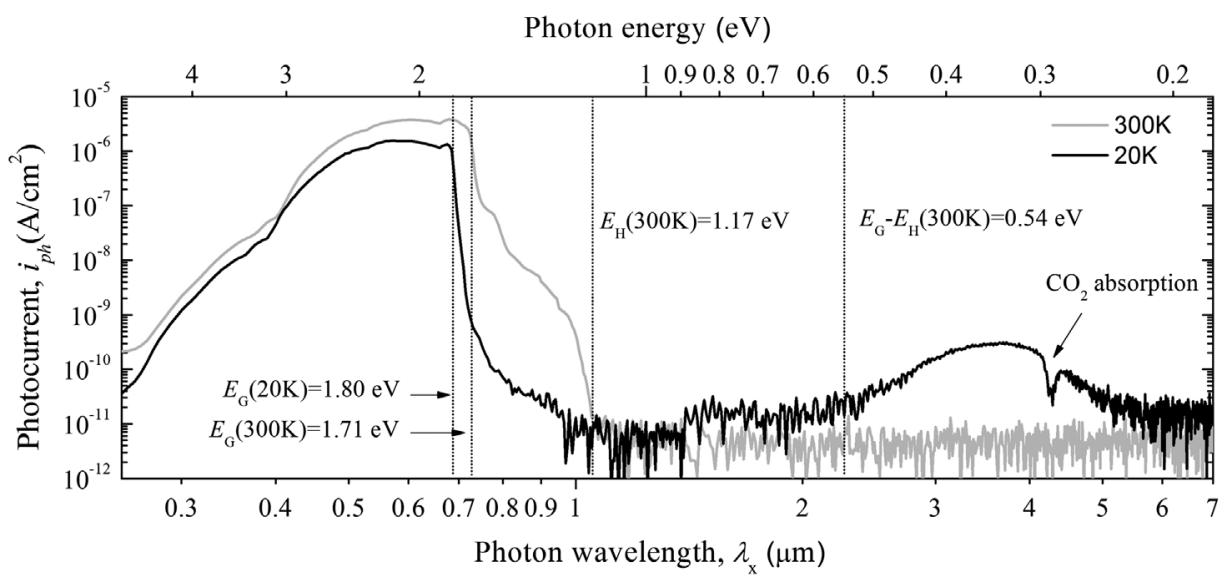

FIG. 4. Spectral photocurrent of the QD IBSC at $300 \mathrm{~K}$ and $20 \mathrm{~K}$. 
is stopped at a position that blocks the light and the lock-in is synchronized to chopper 2). Results are shown in Fig. 4 as a function of the incident photon wavelength $\left(\lambda_{x}\right)$ for two cell temperatures $(300 \mathrm{~K}$ and $20 \mathrm{~K})$. At room temperature $(300 \mathrm{~K})$ the photocurrent detected for photon energies between $E_{H}=1.17 \mathrm{eV}$ and $E_{G}=1.71 \mathrm{eV}$ corresponds to the VB-IB transition labeled 2 in Fig. 1, which has been extensively characterized in previous works [7]. This signal is drastically reduced at low temperatures $(20 \mathrm{~K})$, indicating that the thermal excitation of electrons from the QD states (IB) to the CB has been inhibited. At the same time this thermal excitation is reduced, photocurrent is detected within the photon energy interval of $0.25-0.55 \mathrm{eV}$, which corresponds to the IB-CB transition labeled 3 in Fig. 1. To our knowledge, this is the first time that a QD IBSC cell has been demonstrated to be capable of producing photocurrent when illuminated solely by photons that pump electrons from the IB to the $\mathrm{CB}$ (photon energies below $E_{H}$ ). Notice that this photocurrent does not produce electrical work because the cell is short circuited.

How photocurrent can be extracted from the device by the incidence of photons with energies below $E_{H}$ can be explained by means of the simplified IBSC circuit model in the short-circuit conditions shown in Fig. 5(a). In this circuit, diodes describe the internal generation recombination (GR) between electronic bands (e.g., $D 2$ describes the internal GR between the IB and VB) and their voltages correspond to the difference between the quasi-Fermi levels of those bands. Notice that, in short-circuit conditions, the band diagram of an ideal IBSC with infinite carrier mobility is equal to that in equilibrium; i.e., all quasi-Fermi levels merge together. However, in real devices, the finite mobility of minority carriers produces a quasi-Fermi level split within the device [17] [Fig. 1(b)], enabling internal recombination in short circuit. This effect is accounted for in the circuit of Fig. 5(a) by means of the lumped resistance $R_{s}$, which makes possible that diodes $D 1, D 2$, and $D 3$ become biased in short circuit with a bias current $I_{D i}=I_{0 i}\left[\exp \left(q V_{D i} / k T\right)-1\right]$, where $V_{D i}$ is the diode bias voltage associated to the quasi-Fermi level split, and $I_{0 i}$ is the diode saturation current. Notice that in this simplified model, $I_{0 i}$ accounts for all the possible internal GR mechanisms in the semiconductor (radiative, Auger, or

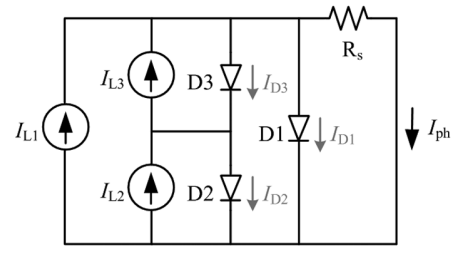

(a)

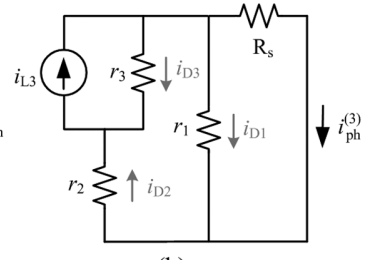

(b)
FIG. 5. (a) Simplified IBSC equivalent circuit in short-circuit conditions. (b) Small-signal model for illumination causing IB to $\mathrm{CB}$ transitions.
Shockley-Read-Hall). A more complex model segregating each type of GR along with considering radiative coupling and shunt resistances could be used [18]. However, the simplified circuit in Fig. 5 will suffice to provide a qualitative explanation of the results of the experiment.

Because of the low amplitude of the photocurrent produced by IB-CB transitions (Fig. 4), the circuit in Fig. 5(a) can be simplified to the small-signal equivalent circuit in Fig. 5(b) when the energy of the incident photons is capable of pumping electrons from the IB to the CB. This circuit is constructed by replacing diodes $D i$ by their equivalent small-signal resistances $r_{i}=d V_{D i} / d I_{D i}=$ $(k T / q)\left(I_{D i}+I_{0 i}\right)^{-1}$. According to this model, the detection of $i_{\mathrm{ph}}^{(3)}$, the mid-IR photocurrent shown in Fig. 4 , is possible due to the existence of noninfinite resistance $r_{2}\left(I_{D 2}\right)$ that connects the VB with the IB, which ultimately depends on the internal GR mechanisms between the VB and IB accounted for by $I_{02}$.

In order to corroborate that the circuit of Fig. 5 actually describes the operation of the QD IBSC, we conducted an additional experiment consisting of measuring the smallsignal IR response $i_{\mathrm{ph}}^{(3)}$ under variable supra-band gap continuous illumination, producing an output photocurrent $I_{\mathrm{ph}}$. The IR response $i_{\mathrm{ph}}^{(3)}$ was measured tuning the lock-in to chopper 1 with the chopper 2 open (Fig. 3). Different $I_{\mathrm{ph}}$ values were obtained by varying the width of the monochromator slits and the MC output wavelength $\lambda_{x}$ in the range of $250-640 \mathrm{~nm}$. $I_{\mathrm{ph}}$ was measured by momentarily switching the lock-in to chopper 2 and closing chopper 1 . Experimental results are shown in Fig. 6 together with the results of the application of the circuital model for $i_{L 3}=2.44 \times 10^{-8} \mathrm{~A} / \mathrm{cm}^{2}, \quad R_{s}=51.8 \mathrm{k} \Omega \mathrm{cm}^{2}, \quad I_{01}=$ $2.64 \times 10^{-80} \mathrm{~A} / \mathrm{cm}^{2}, I_{02}=4.65 \times 10^{8} \times I_{01}$, and $I_{03}=$ $20.19 \times I_{02}$. Notice that $i_{\mathrm{ph}}^{(3)}$ was found to depend exclusively on $I_{\mathrm{ph}}$ but not on the supra-band gap MC wavelength.

In Fig. 6 we observe three different regimes that can be explained by the circuital model of Fig. 5. (1) For

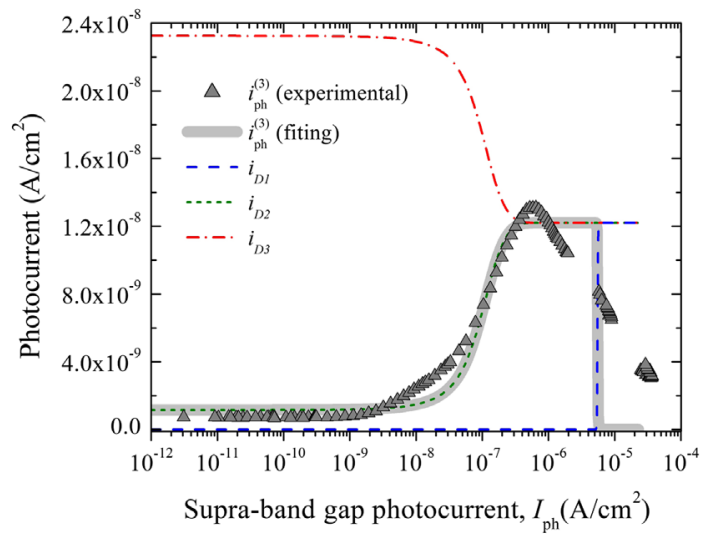

FIG. 6 (color online). IR small-signal photocurrent in the QD IBSC as a function of supra-band gap photocurrent. 


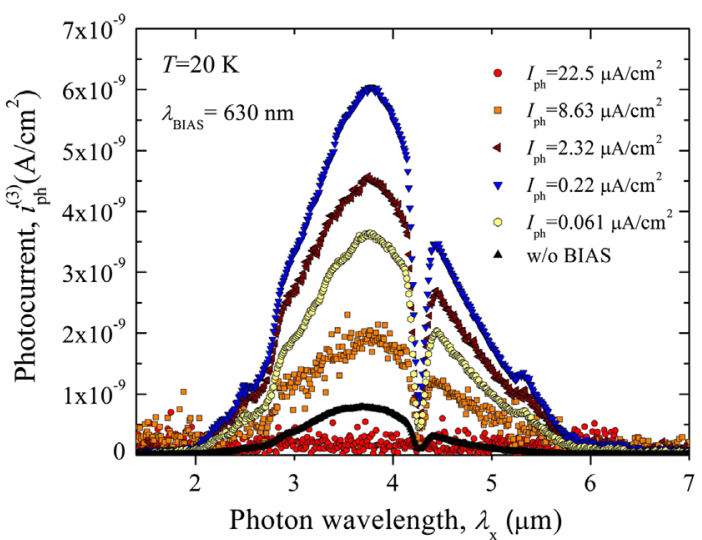

FIG. 7 (color online). Spectral photocurrent of the QD IBSC as a function of supra-band gap photocurrent.

$I_{\mathrm{ph}}<10^{-9} \mathrm{~A} / \mathrm{cm}^{2}, i_{\mathrm{ph}}^{(3)}$ is low because diode $D 2$ operates near short circuit and has a high equivalent resistance $r_{2} \approx k T / q I_{02}$, which indicates that the IR photocurrent $i_{L 3}$ is mostly recombined internally in $D 3$. Notice that a very low saturation current in $D 2\left(I_{02}\right)$ would make $i_{\mathrm{ph}}^{(3)}$ undetectable. (2) For $10^{-9}<I_{\mathrm{ph}}<5 \times 10^{-7} \mathrm{~A} / \mathrm{cm}^{2}, D 2$ enters the direct-bias regime, $r_{2}$ is reduced, and consequently, $i_{\mathrm{ph}}^{(3)}$ increases. (3) For $I_{\mathrm{ph}}>5 \times 10^{-7} \mathrm{~A} / \mathrm{cm}^{2}$, the diode $D 1$ enters in the direct-bias regime, recombining most of $i_{L 3}$, and leading to the reduction of $i_{\mathrm{ph}}^{(3)}$. In the simplified model represented by the circuit in Fig. $5, i_{\mathrm{ph}}^{(3)}$ sharply goes to zero due to the ideal characteristic of diode $D 1$. In the real device this transition is softened, probably due to the existence of additional recombination mechanisms between $\mathrm{CB}$ and VB and due to the indirect photofilling of the QDs (affecting to the QD absorption), none of them are accounted for in this simplified model.

A final experiment was conducted in order to corroborate that the dependence with $I_{\mathrm{ph}}$ shown in Fig. 6 actually corresponds to variations in the IR spectral response of the QD IBSC. For that, the spectral photocurrent in the IR was also measured as a function of supra-band gap $I_{\text {ph }}$ photocurrent. Results are shown in Fig. 7. The IR spectral photocurrent increases with $I_{\mathrm{ph}}$ until a certain threshold (between 0.06 and $2.32 \mu \mathrm{A} / \mathrm{cm}^{2}$ ), from which it decreases again until it becomes undetectable at $I_{\mathrm{ph}} \approx 20 \mu \mathrm{A} / \mathrm{cm}^{2}$. This is in agreement with the experiments shown in Fig. 6.

Therefore, results shown in Figs. 6 and 7 validate our initial hypothesis that the mid-IR response of the QD IBSC (Fig. 4) is attributed to a non-negligible GR between the IB and the VB through diode $D 2$, which has a relatively high saturation current.

In summary, we have shown for the first time that a QD IBSC is capable of producing photocurrent when illuminated solely with photons producing the IB-CB transition. This current is measurable thanks to the non-negligible GR between VB and IB. By exploring the bias dependence of this photocurrent, we have demonstrated that the simplest circuital model of an IBSC is able to describe qualitatively the experimental results, demonstrating that it is in agreement with the operation principles of an IBSC.

This work has been funded by the European Commission and NEDO through the collaborative Project NGCPV (Grant No. 283798).

[1] A. Luque and A. Martí, Phys. Rev. Lett. 78, 5014 (1997).

[2] R. W. Peng, M. Mazzer, and K. W. J. Barnham, Appl. Phys. Lett. 83, 770 (2003).

[3] A. Marti, L. Cuadra, and A. Luque, in Proceedings of the 28th IEEE Photovoltaic Specialist Conference, 2000 (IEEE, Anchorage, 2000), pp. 940-943.

[4] K. M. Yu, W. Walukiewicz, J. Wu, W. Shan, J. W. Beeman, M. A. Scarpulla, O. D. Dubon, and P. Becla, Phys. Rev. Lett. 91, 246403 (2003).

[5] A. Luque, A. Martí, E. Antolín, and C. Tablero, Physica (Amsterdam) 382B, 320 (2006).

[6] I. Ramiro, A. Martí, E. Antolin, and A. Luque, IEEE J. Photovoltaics 4, 736 (2014).

[7] A. Luque, A. Martí, C. Stanley, N. López, L. Cuadra, D. Zhou, J. L. Pearson, and A. McKee, J. Appl. Phys. 96, 903 (2004).

[8] A. Luque, A. Marti, and L. Cuadra, IEEE Trans. Electron Devices 48, 2118 (2001).

[9] A. Marti, E. Antolin, C. R. Stanley, C. D. Farmer, N. Lopez, P. Diaz, E. Canovas, P. G. Linares, and A. Luque, Phys. Rev. Lett. 97, 247701 (2006).

[10] R. Tamaki, Y. Shoji, Y. Okada, and K. Miyano, Appl. Phys. Lett. 105, 073118 (2014).

[11] I. Ramiro, E. Antolin, P. Linares, E. Lopez, I. Artacho, A. Datas, A. Marti, A. Luque, M. Steer, and C. Stanley, in Proceedings of the IEEE 40th Photovoltaic Specialist Conference (PVSC), 2014 (IEEE, Denver, 2014), pp. 3251-3253.

[12] P. G. Linares, A. Marti, E. Antolin, C. D. Farmer, I. Ramiro, C. R. Stanley, and A. Luque, Sol. Energy Mater. Sol. Cells 98, 240 (2012).

[13] S. Sauvage, P. Boucaud, F. H. Julien, J.-M. Gérard, and V. Thierry-Mieg, Appl. Phys. Lett. 71, 2785 (1997).

[14] K. A. Sablon, J. W. Little, V. Mitin, A. Sergeev, N. Vagidov, and K. Reinhardt, Nano Lett. 11, 2311 (2011).

[15] A. Martí, E. Antolín, E. Cánovas, N. López, P. Linares, A. Luque, C. Stanley, and C. Farmer, Thin Solid Films 516, 6716 (2008).

[16] E. Antolín, A. Martí, C. D. Farmer, P. G. Linares, E. Hernández, A. M. Sánchez, T. Ben, S. I. Molina, C. R. Stanley, and A. Luque, J. Appl. Phys. 108, 064513 (2010).

[17] K. Yoshida, Y. Okada, and N. Sano, Appl. Phys. Lett. 97, 133503 (2010).

[18] A. Luque, A. Marti, C. Stanley, N. Lopez, L. Cuadra, D. Zhou, J. L. Pearson, and A. McKee, J. Appl. Phys. 96, 903 (2004). 